\title{
Integrated analysis of long non-coding RNAs and mRNAs reveals the regulatory network of maize seedling root responding to salt stress
}

\author{
Peng Liu, Yinchao Zhang, Chaoying Zou, Cong Yang, Guangtang Pan, Langlang Ma and Yaou Shen* (i)
}

\begin{abstract}
Background: Long non-coding RNAs (IncRNAs) play important roles in response to abiotic stresses in plants, by acting as cis- or trans-acting regulators of protein-coding genes. As a widely cultivated crop worldwide, maize is sensitive to salt stress particularly at the seedling stage. However, it is unclear how the expressions of protein-coding genes are affected by non-coding RNAs in maize responding to salt tolerance.

Results: The whole transcriptome sequencing was employed to investigate the differential IncRNAs and target transcripts responding to salt stress between two maize inbred lines with contrasting salt tolerance. We developed a flexible, user-friendly, and modular RNA analysis workflow, which facilitated the identification of IncRNAs and novel mRNAs from whole transcriptome data. Using the workflow, 12,817 IncRNAs and 8,320 novel mRNAs in maize seedling roots were identified and characterized. A total of 742 IncRNAs and 7,835 mRNAs were identified as salt stress-responsive transcripts. Moreover, we obtained 41 cis- and 81 trans-target mRNA for 88 of the IncRNAs. Among these target transcripts, 11 belonged to 7 transcription factor (TF) families including $b H L H, C 2 H 2$, Hap3/NF-YB, HAS, MYB, WD40, and WRKY. The above 8,577 salt stress-responsive transcripts were further classified into 28 modules by weighted gene co-expression network analysis. In the salt-tolerant module, we constructed an interaction network containing 79 nodes and 3081 edges, which included 5 IncRNAs, 18 TFs and 56 functional transcripts (FTs). As a trans-acting regulator, the IncRNA MSTRG.8888.1 affected the expressions of some salt tolerance-relative FTs, including protein-serine/threonine phosphatase $2 \mathrm{C}$ and galactinol synthase 1 , by regulating the expression of the bHLHTF.

Conclusions: The contrasting genetic backgrounds of the two inbred lines generated considerable variations in the expression abundance of IncRNAs and protein-coding transcripts. In the co-expression networks responding to salt stress, some TFs were targeted by the IncRNAs, which further regulated the salt tolerance-related functional transcripts. We constructed a regulatory pathway of maize seedlings to salt stress, which was mediated by the hub IncRNA MSTRG.8888.1 and participated by the bHLHTF and its downstream target transcripts. Future work will be focused on the functional revelation of the regulatory pathway.
\end{abstract}

Keywords: Maize, Salt stress, IncRNA, TFs, Protein-coding transcript, WGCNA

*Correspondence: shenyaou@sicau.edu.cn

State Key Laboratory of Crop Gene Exploration and Utilization

in Southwest China, Maize Research Institute, Sichuan Agricultural

University, Chengdu 611130, P. R. China

\section{Background}

Globally, over 831 million hectares of the land have been influenced by salinity (http://www.fao.org), which reduces the water availability, inhibits the growth and development, and causes the decreased yield in crops [1]. The main effects of salt stress in plant are as follows: (1) High ambient concentrations of salt increase the cell 
water potential, change the cell osmotic pressure, hence cause the loss of cell water, and result in physiological drought [2]. (2) The accumulated ions break cell ion balance and thus cause ion toxicity, due to the nutrient imbalance in the cytosol. (3) Salt stress disturbs reactive oxygen species (ROS) and induces oxidative stress [2-4]. Maize (Zea mays L.) is a widely cultivated crop worldwide, and it is sensitive to salt stress particularly at the seedling stage [5]. Understanding how maize responds to salt stress could help to develop salt-tolerant maize lines for maize breeding. In maize, large numbers of proteincoding genes involved in salt stress have been reported in previous studies. Overexpression of the Suaeda salsa $\mathrm{Na}^{+} / \mathrm{H}^{+}$antiporter gene $(S s N H X 1)$ in maize enhances the salt tolerance of the transgenic maize [6]. ZmSnRK2.11 is a potential negative regulator involved in maize salt stress, which is up-regulated by high salinity. Overexpression of $Z m S n R K 2.11$ in Arabidopsis caused the salt sensitivity phenotypes, including increased rate of water loss, reduced relative water content, and delayed stoma closure [7]. The high-affinity potassium transporter gene $(H K T 1)$ affects $\mathrm{K}^{+}$and $\mathrm{Na}^{+}$transports in roots and shoots, regulates $\mathrm{K}^{+} / \mathrm{Na}^{+}$homeostasis, and thus improves the tolerance to $\mathrm{Na}^{+}$stress in maize [8]. The $H A K$ family ion transporter ZmHAK4 confers the shoot $\mathrm{Na}^{+}$exclusion and salt tolerance in maize by retrieving $\mathrm{Na}^{+}$from xylem sap [9]. In addition, some transcript factors (TFs) are also associated with salt stress response in maize. For instance, $Z m M Y B 3 R$ positively regulates maize tolerance to salt stress via an ABA-dependent pathway [10]. Some other TF families including $H S F$, $N A C, W R K Y$, and $b Z I P$ have been reported to participate the response to salt stress [11-15]. Furthermore, several signaling-related genes and plant hormones-related genes were proven to correlate with maize salt tolerance [16-19]. However, the molecular regulatory networks of these genes have not been fully elucidated. Especially, it is still obscure how the expression of protein-coding genes is affected by non-coding RNA in maize [20].Long noncoding RNA (lncRNA) is a type of non-coding RNA that has $\geq 200$ nucleotides in length. Based on their genomic localizations relative to protein-coding genes, lncRNAs are mainly classified into long intergenic ncRNAs (lincRNAs), long intronic noncoding RNAs (intron-lncRNAs), and natural antisense transcripts (NATs) [21]. LncRNAs can affect gene expression by acting as cis- or transacting regulators $[22,23]$. Li et al. identified more than 1,700 high-confidence lncRNAs among 20,163 putative lncRNAs in maize at a genome-wide level [24]. HuancaMamani et al. identified in a hyper-arid maize line 1,710 putative lncRNAs responsive to the combined stress of salt and boron, which showed an unusual higher expression relative to protein-coding genes under the stress conditions [25]. Lv et al. identified 1,077 differentially expressed lncRNAs in maize, including 509 transposable element (TE)-lncRNAs. The construction of co-expression networks further revealed 39 lncRNAs as major hubs that respond to abiotic stress, among which 18 were derived from TEs [26]. However, the reports on saltresponsive lncRNAs are still being discovered in maize.

By using two maize inbred lines with contrasting salt tolerance, we constructed 28 total RNA libraries across four stages of salt treatment for whole transcriptome sequencing. To accurately identify and characterize lncRNAs and their targets responding to salt stress, we developed a lncRNA and novel mRNA identification pipeline named NLncCirSmk by integrating different current methods. NLncCirSmk is based on the snakemake workflow management system, an open-source tool for creating reproducible and scalable data analyses [27]. NLncCirSmk is freely available on GitHub (https:// github.com/Alipe2021/NLncCirSmk). By employing NLncCirSmk, the researchers can simultaneously identify differentially expressed lncRNAs, circRNAs and novel mRNAs from the whole transcriptome sequencing data.

\section{Results \\ High-throughput sequencing and analysis workflow developing}

The lines L2010-3 and BML1234 were selected from an association panel of 330 maize inbred lines through a salt stress-tolerance test. Under salt stress, the salt-sensitive line BML1234 showed a more serious growth inhibition relative to the salt-tolerant line L2010-3, including decreased plant height and reduced biomass [28]. Under salt treatment, the two lines with contrasting salt tolerance were subjected to the construction of whole transcriptome libraries. Specifically, a total of 28 samples were collected from the tolerant line (L2010-3) and the sensitive line (BML1234) under CK $(0,6,18$, and $36 \mathrm{~h})$ and salt treatment $(6,18$, and $36 \mathrm{~h})$, respectively, with two biological repetitions. To improve the efficiency of bioinformatic analysis, we developed a flexible, user-friendly, and modular RNA analysis workflow, named Novel mRNA, LncRNA, and CircRNA Analysis Snakemake Workflow (NLncCirSmk). It is based on the package management software Conda and the workflow management system Snakemake. The present workflow includes a complete pipeline for novel mRNA, lncRNA, and circRNA (developing) analysis (Fig. 1). NLncCirSmk starts with the quality control of raw FASTQ files from paired sequencing data, going through optional trimming and rRNA filtering, alignment and assembly, identification of lncRNAs and novel mRNAs, and expression analysis. The workflow supports parallel computing and can greatly 
improve the speed of data processing. The source code of NLncCirSmk is available on GitHub (https://github.com/ Alipe2021/NLncCirSmk).

\section{QC, rRNA filtering, transcripts assembly, and expression analysis}

By performing high-throughput RNA-Seq, a total of 529.5G raw data were generated from the 28 whole transcriptome libraries. After removing low-quality reads, approximate $510.0 \mathrm{G}$ clean bases were obtained. Among them, $93.01 \%$ reads had quality scores at the Q30 level (ratio of error rate $\leq 0.1 \%$ ). By mapping the clean reads to the rRNA database, $1.89 \%$ to $9.70 \%$ clean reads were identified as rRNAs and were then filtered out (Table S1,Fig. 1). The remaining rRNA-free reads were aligned to the maize reference genome (B73 RefGen_v4) [29], with the alignment ratio ranging from $33.56 \%$ to 66.33\%. After transcriptome assembly, an integrated transcript set including 206,712 transcripts was reconstructed from all the 28 RNA-seq datasets. The sequencing and mapping statistics were summarized in Table S1.

Correlation analysis showed that different biological repetitions had a high consistency and were clustered together (Fig. 2A). PCA displayed that more than $43 \%$ of the variability in gene expression abundance among the samples were explained by the first three principal components (PCs) (Fig. 2B). The overall gene expression levels of the 28 samples were evidently clustered into four different groups by different materials and treatment conditions. Especially, the obvious difference was observed between two contrasting lines. The samples from the salt-sensitive line BML1234 fell in the negative direction, whereas the samples from the salt-tolerant line L2010-3 resided in the positive direction of the $\mathrm{PC} 1$ axis. In the PC2 axis, the samples under salt treatment fell in the positive direction of the axis, and majority of the samples under normal conditions fell in the negative direction of the axis (Fig. 2C). Moreover, the samples under normal conditions were mainly situated in the positive direction of the PC3 axis for both lines (Fig. 2D). It revealed a remarkable difference in gene expression under the salt stress and normal condition.

\section{Identification and characterization of IncRNAs and novel mRNAs}

After filtering out the unqualified transcripts (length $<200$, exon numbers $<2$, and coverage $<3$ ), 1,501 , 696, 20,993, and 1,263 transcripts with class_code of "i", "o", "u", and "x" were detected, respectively, by comparing their genomic locations and directions to the reference transcripts. By removing potential protein coding transcripts (PCTs), a total of 12,817 common transcripts were defined as lncRNAs, including 422 known lncRNAs and 12,395 novel lncRNAs (Fig. 3A). Among them, 833 (6.50\%), $11,417(89.08 \%)$, and 567 (4.42\%) belonged to intronic-lncRNAs, lincRNAs, and antisense-lncRNAs, respectively (Fig. 4A,4B). In addition, 8,320 transcripts were identified as novel PCTs (Fig. 3B). According to the homologous annotations, 997 (12.39\%) novel PCTs were involved in signal transduction, whereas 698 (8.67\%) novel PCTs were related to posttranslational modification, protein turnover, and chaperones. The majority $(1,867)$ of these novel PCTs were unknown transcripts (TableS2,FigureS1). In comparison with PCTs, 12,201 $(95.19 \%)$ of the novel lncRNAs had fewer $(<3)$ exons and 11,755 (91.71\%) had shorter (<2000 bp) tags (Fig. 3C,3D). The above findings were consistent with the previous studies in maize [24], Cleistogenes songorica [30], Carya cathayensis [31], and duckweed [32]. The detailed flowchart for identifying lncRNAs and novel PCTs was shown in Fig. 1.

\section{Validation of the assembled transcripts by qRT-PCR}

To validate the expression levels of the assembled transcripts from RNA-seq, six transcripts (MSTRG.26461.5, MSTRG.28025.2, MSTRG.54905.9, MSTRG.61639.4, Zm00001d001353_T001 and Zm00001d021924_T001) were randomly subjected to expression examination by qRT-PCR. The Pearson's correlation coefficient between RNA-Seq and qRT-PCR was calculated. The expression levels of these transcripts from RNA-seq data were significantly correlated with those using qRT-PCR $\left(R^{2} \geq 0.6112, P<0.001\right)$ (Table S3, Figure S2), indicating that the expression profile based on RNA-seq data was reliable in the present study.

\section{LncRNAs and $\mathrm{PCTs}$ responding to salt stress}

The differentially expressed lncRNAs (DELs) were identified according to the criteria: $|\log 2 \mathrm{FC}|>2, P$-adjust $<0.01$.

\footnotetext{
(See figure on next page.)

Fig. 1 Workflow of bioinformatic analysis in our research. The figure shows the process of bioinformatic analysis in this study. The orange box illustrates the criteria for data quality control, the identification criteria for non-coding RNAs are listed in the pale green box, and the identification criteria for novel protein-coding RNAs are listed in the yellow box. The identification of IncRNAs and novel mRNAs are shown in the green rectangle. Differential expression analysis and gene co-expression analysis are shown in the purple rectangle. GO and KEGG analysis for mRNAs are displayed in the two cyan rounded rectangles. The gene regulation network shown in an irregular graph was constructed by integrating the results of differential expression analysis, co-expression analysis, and target prediction of IncRNAs
} 


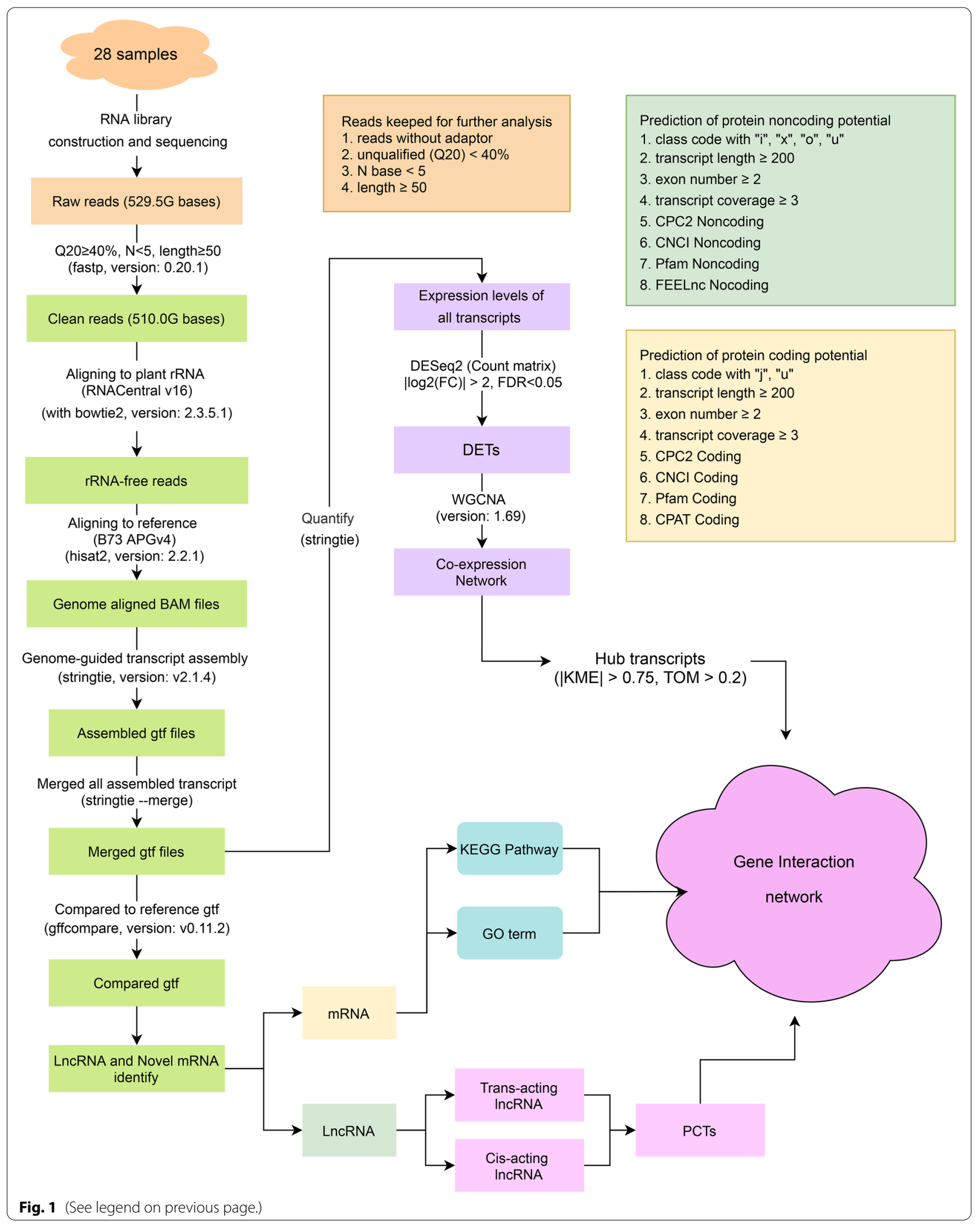




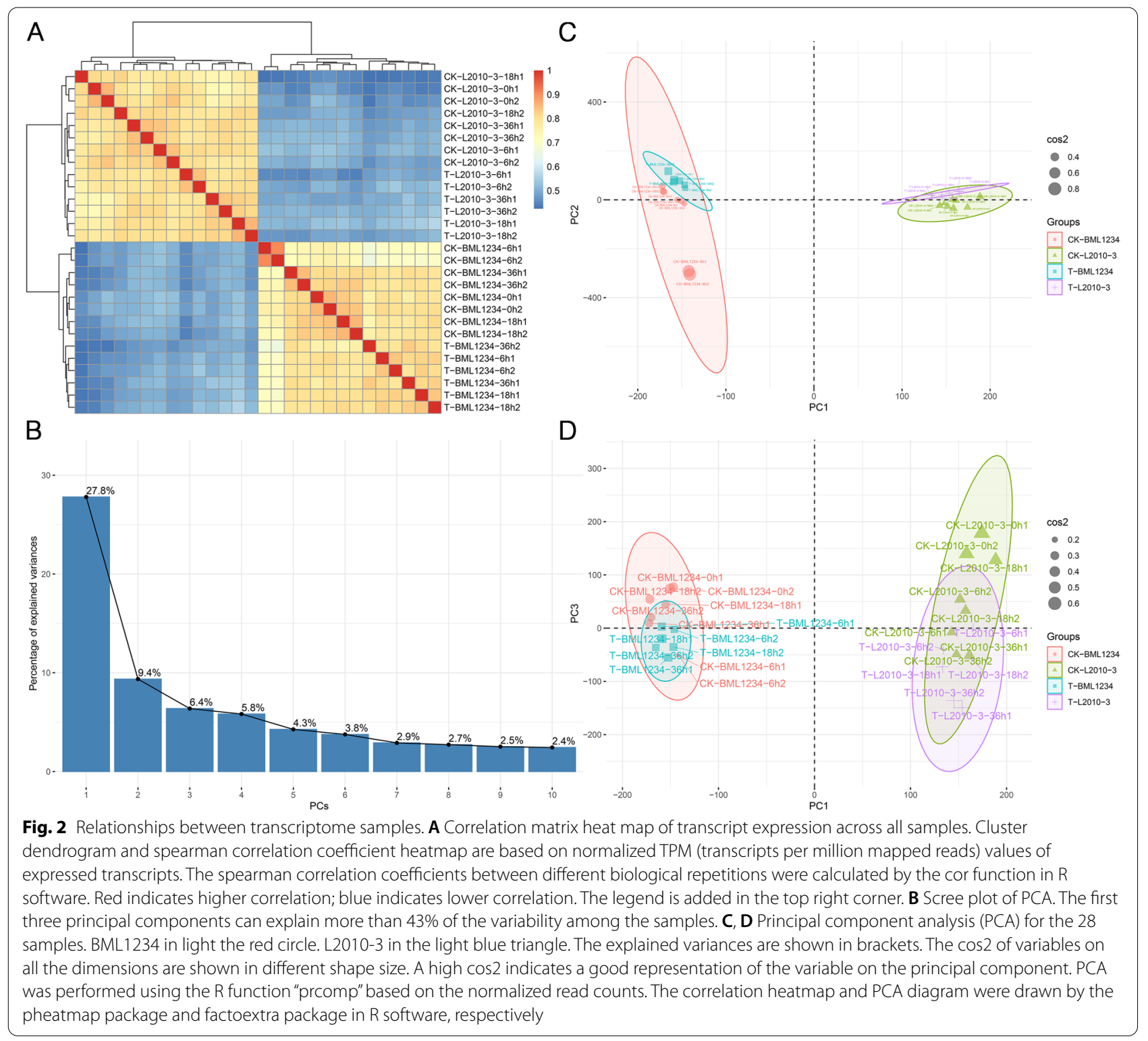

In the salt-sensitive line BML1234, 592 (517 upregulated and 75 downregulated), 47 (26 upregulated and 21 downregulated), and 56 (24 upregulated and 32 downregulated) DELs were detected at 6,18 , and $36 \mathrm{~h}$ under salt stress, respectively (Fig. 5A,Fig. S3A-C). In the salt-tolerance line L2010-3, 53 (31 upregulated and 22 downregulated), 89 ( 35 upregulated and 54 downregulated), and 65 (24 upregulated and 41 downregulated) DELs were detected at each salt treatment stage (Fig. 5A,Fig. S3DF). Among these DELs, 598 and 114 were specifically responsive to salt stress in BML1234 and L2010-3, respectively, whereas 30 DELs were common between the two lines (Fig. 5B). The DELs in BML1234 between normal and salt stress conditions at $6 \mathrm{~h}$ was much more than those in the other samples, implicating that the response of lncRNAs to salt stress mainly occurred at the early stage of the salt treatment in the sensitive line. Besides, the expression of lncRNAs in the sensitive material was more easily affected by the salt stress compared with the tolerant line. By comparing the IncRNA expression levels at a given treatment stage between the two lines, we identified 3,038, 2,795, and 2,792 DELs at 6, 18, and $36 \mathrm{~h}$ of salt treatment (Fig. 5A,Fig. S3G-I). Similarly, a total of 20,107 differentially expressed transcripts (DETs) (13,911 known mRNAs, 4,511 lncRNAs, and 1,685 novel mRNAs) were found between the two lines under normal conditions. These suggested that the contrasting genetic 


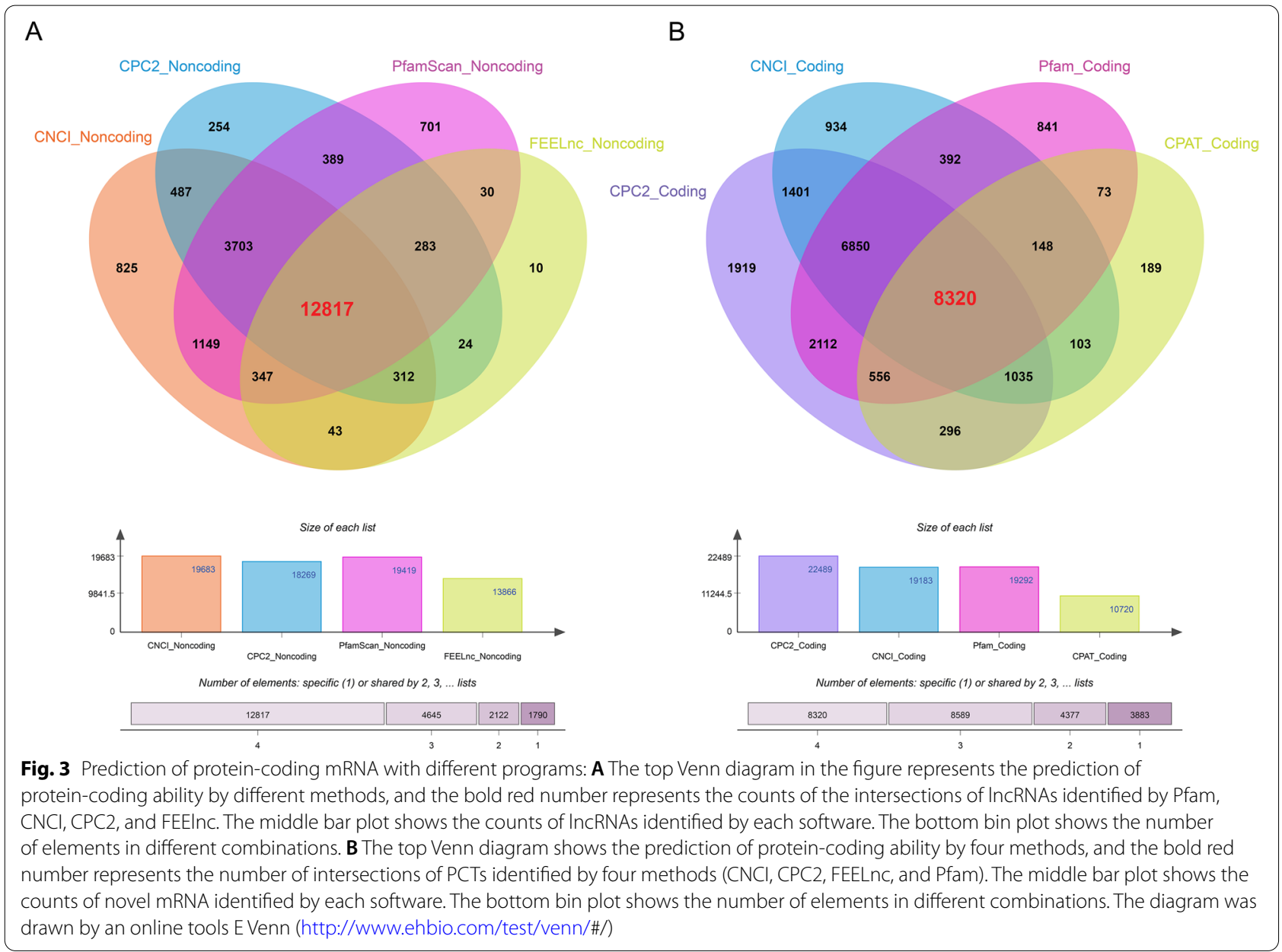

backgrounds of the two lines generated considerable variations in transcript expression abundance.

In addition, we obtained a total of 7,835 differentially expressed mRNAs (DEMs), which presented different salt-stress responses between the two lines, involving 5,672 and 1,753 specific DEMs in BML1234 and L20103 , respectively. Among the 7,835 DEMs, 821 were newly identified PCTs, involving 507 and 272 specific novel PCTs in BML1234 and L2010-3, respectively (Fig. 5C). Furthermore, 998 DEMs were defined as TFs, among which $C 2 H 2, W D 40, M Y B, P H D$, and $b Z I P$ families were the top five largest TF families, individually including $151,126,81,76$, and 38 DEMs (Table S4). At each comparison stage between $\mathrm{CK}$ and treatment conditions, more differentially expressed TFs (DE-TFs) were detected in BML1234 than in L2010-3 (Fig. 5D).

\section{Predicted IncRNA targets responding to salt stress}

To further address the roles of the 742 potential salt stress-responsive DELs, we identified from all the DEMs the potential cis- and trans- target transcripts. In results,
41 DEMs were located between the $100 \mathrm{~kb}$ upstream and downstream of the 742 DELs and were significantly correlated (Pearson correlation coefficient, $|\mathrm{PCC}|>0.6$, $P$-value $<0.05)$ with their corresponding lncRNAs, which were thereby defined as the cis-regulated target transcripts (Table S5). These 41 cis-transcripts were regulated by 35 regulatory DELs. Meanwhile, 81 DEMs including 5 novel mRNAs with the free energy $<-0.2,|\mathrm{PCC}|>0.8$, and $P$-value $<0.01$ were identified as the trans-target transcripts of 58 DELs (Table S6). In total, 168 lncRNAmRNA pairs including 123 trans- and 45 cis- pairs were detected, which were speculated to be involved in salt tolerance in maize seedlings. These target transcripts were categorized into 17 COGs categories and the top 5 categories were annotated as "function unknown", "transcription", "intracellular trafficking, secretion, and vesicular transport", "inorganic ion transport and metabolism", and "translation, ribosomal structure and biogenesis". In addition, 11 transcripts belonging to $7 \mathrm{TF}$ families were detected, including $\mathrm{C} 2 \mathrm{H} 2$ (Zm00001d016139_T001 and Zm00001d049767_T001), HAS (Zm00001d044281_T003 


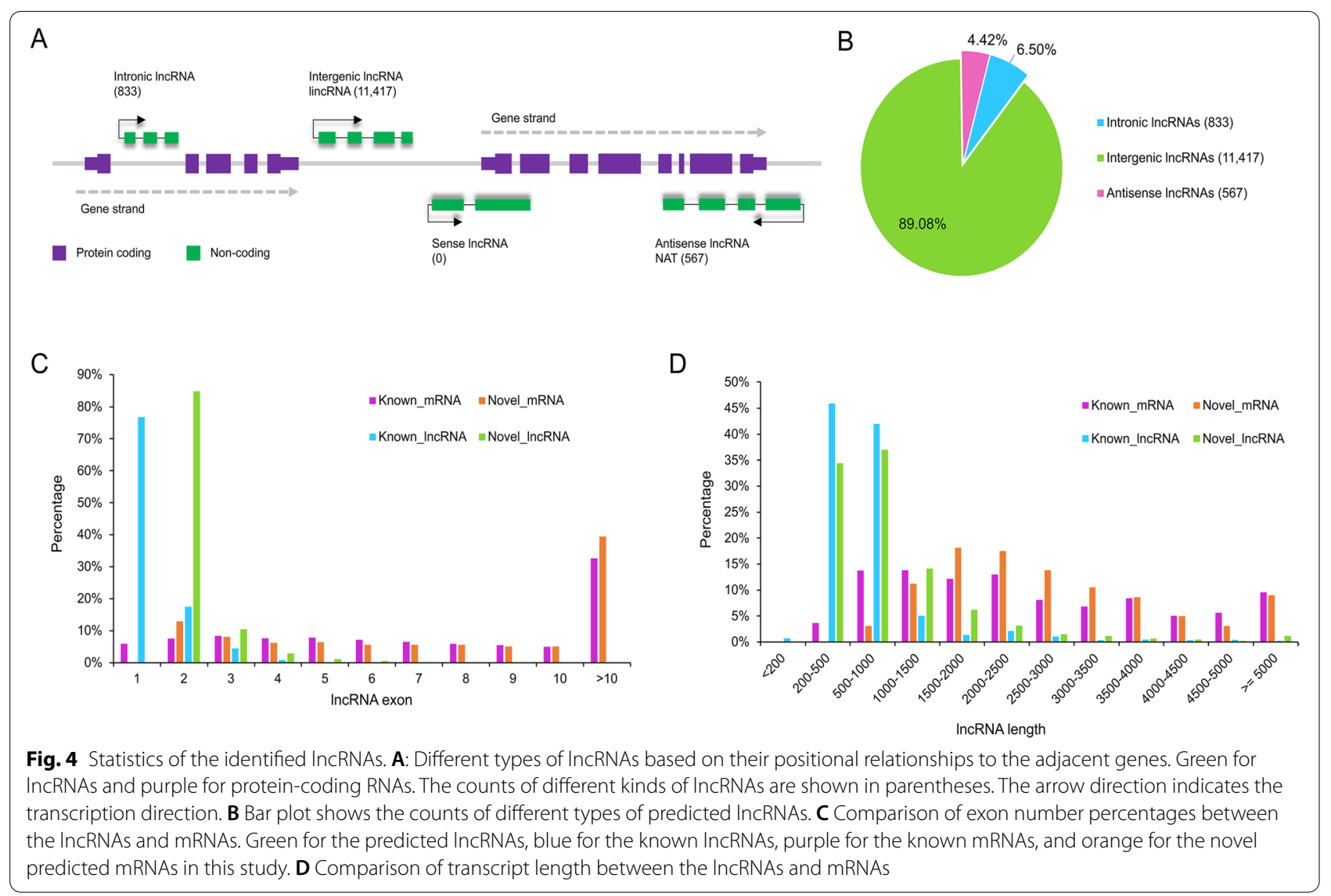

and Zm00001d044283_T005), (Zm00001d032328_T005), (Zm00001d011691 T002, Zm00001d044281 T003, and Zm00001d044283_T005), WD40-like (Zm00001d011920_T002, Zm00001d040038_T003, and Zm00001d046587_T028), WRKY (Zm00001d007329_ T001), and bHLH families (Zm00001d043706_T001). These TFs were previously reported to respond to salt stress, growth and development in plants [33-37]. KEGG enrichment analysis indicated that "RNA polymerase", "oxidative phosphorylation", and "protein export" pathways were significantly enriched with the target transcripts (Table S7). GO analysis uncovered 11 (integral component of plasma membrane, DNA-directed RNA polymerase III complex, and others), 42 (calcium-transporting ATPase activity, cation-transporting ATPase activity, and others), and 14 (single fertilization, ATP hydrolysis coupled transmembrane transport, and others) terms as the most significantly enriched GO terms in cellular component (CC), molecular function (MF), and biological process (BP), respectively (Table S8).

\section{Salinity stress-responsive modules in WGCNA}

Co-expression modules have been used to exhibit the interaction relationships between different functionassociated genes [38, 39]. In total, 8,577 DETs including 7,835 DE-PCTs and 742 DELs were used for constructing the co-expression modules via WGCNA (Table S9). The soft-threshold power of $\beta$ was determined as 4 (Fig. S4) when the scale-free topology index was 0.95 . In total, 28 distinct modules were built with the parameters $($ deepSplit $=2$ and minModuleSize $=30)$, which were labelled with different colors (Fig. 6A). The number of DETs in each module ranged from 37 to 3,237 and 6,748 (83.28\%) DETs were classified into the top ten modules. Moreover, 621 (86.73\%) DELs were clustered into the blue, turquoise, black, yellow, red, and brown modules. To identify the biological function of the DELs in each co-expressed module, we executed KEGG pathway and GO enrichment analyses. The transcripts in the turquoise, red, and brown modules were significantly (FDR $<0.05)$ enriched in 3 ("ribosome", "proteasome", and "ribosome biogenesis in eukaryotes"), 4 ("fatty acid elongation", "cutin, suberine and wax biosynthesis", "biosynthesis of secondary metabolites", and "linoleic acid metabolism"), and 10 ("metabolic 


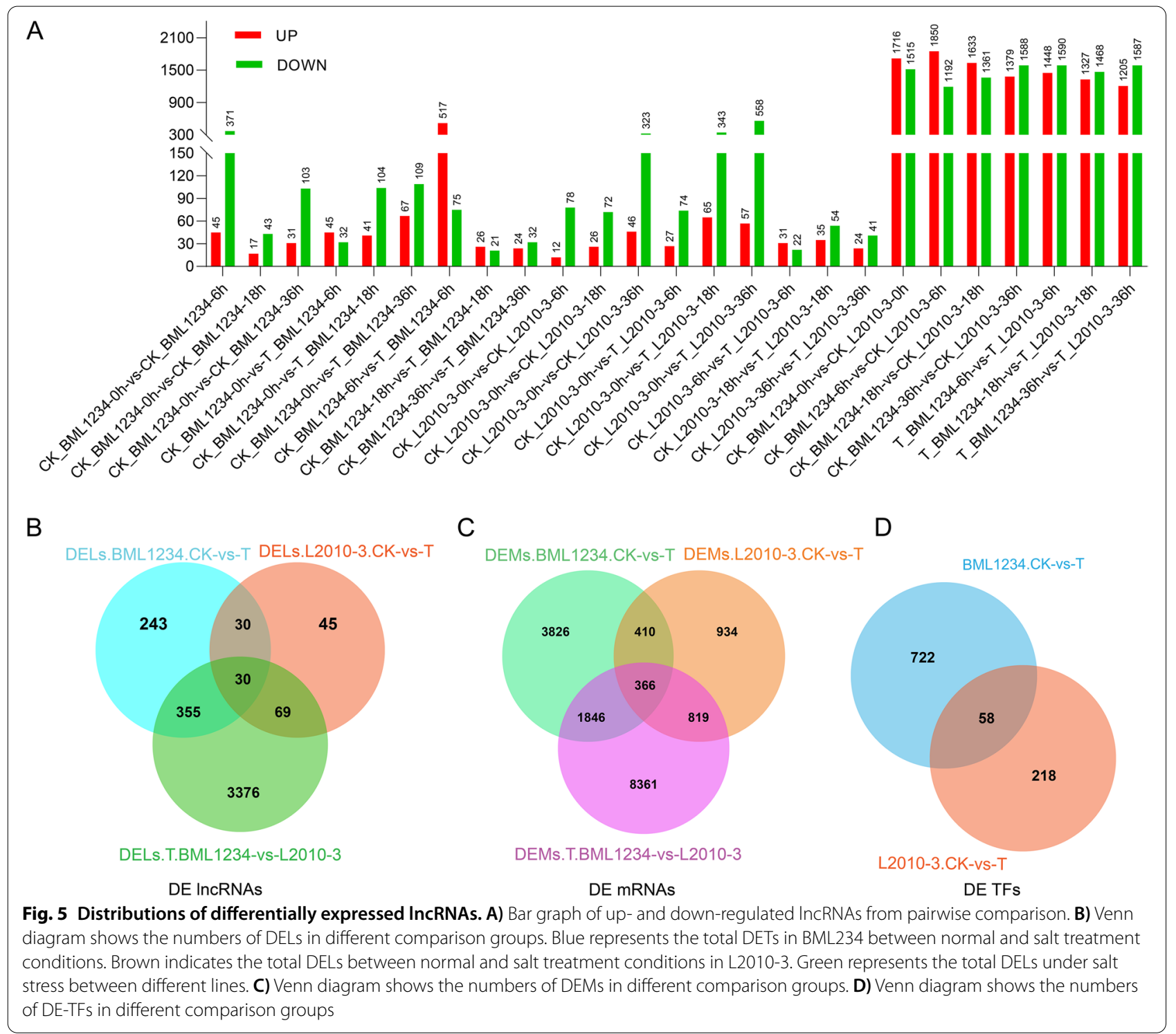

pathways", "plant hormone signal transduction", and others) pathways, respectively. GO analysis showed that 128 ribosome-relevant terms, 88 abiotic stimulus response-related terms, 72 transferase activity-associated terms, and 4 glyoxylate cycle-relevant terms were significantly $(F D R<0.05)$ enriched in the turquoise, red, brown, and yellow modules, respectively. Previous studies reported that plant response to salt stress involves abiotic stimulus response and transferase participation $[19,40]$. Therefore, we further focused on the red and brown modules to identify the hub transcripts. The KME of the transcripts in these modules were calculated by the signedKME function in $\mathrm{R}$ package. In total, 5 lncRNAs (MSTRG.13504.1, MSTRG.16772.1, MSTRG.58725.1, MSTRG.6043.1, and MSTRG.8888.1) and 231 PCTs were identified as the hub transcripts $(|\mathrm{KME}|>0.75, \mathrm{TOM}>0.2)$ in brown module. Interestingly, 14 PCTs were the target transcripts of 5 DELs in the hub transcript set, including the $b H L H$ TF (Zm00001d043706_T001)/ MSTRG.8888.1 pair. Meanwhile, 85 hub transcripts including 2 lncRNAs (MSTRG.62146.4 and MSTRG.68516.1) and 83 mRNAs were detected in the red module, of which 25 transcripts were significantly enriched in the results of GO analysis.

\section{Regulatory network mediated by IncRNAs and their target TFs}

Since some TFs were identified as the targets of lncRNAs in the brown module, we further constructed the 


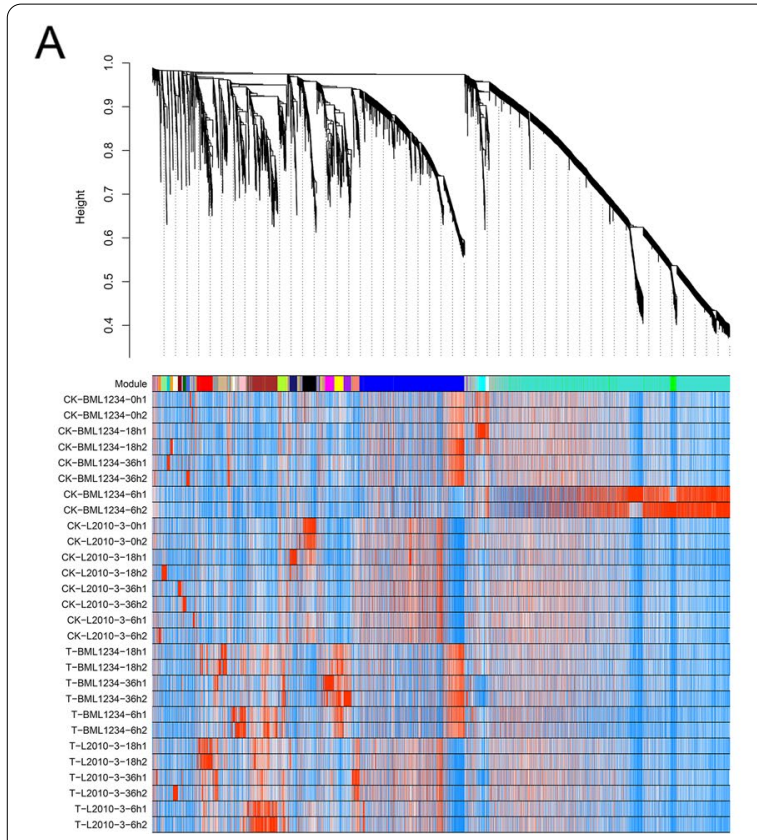

$\mathrm{B}$

Fig. 6 Co-expression modules of DETs and network dynamics in response to salt stress in maize seedling. A) Transcripts hierarchical clustering tree of different modules. Each major tree branch stands for one module, each leaf in the tree represents one transcript, different modules are labelled with different colors. B) Detailed network dynamics of nodes in brown module. Dark blue ellipses represent the top five connected mRNAs for each IncRNA, pink ellipse displays one of top five connected mRNAs to the $b H L H$, dark purple ellipses stand for common mRNAs in top five connected mRNAs of $b H L H$ and IncRNAs, light blue shows other mRNAs

regulatory networks of salinity response mediated by lncRNAs and their target TFs. In the brown module, a network with 79 nodes and 3,082 edges was established, which contained 5 DELs, 18 TFs, 3 novel mRNAs, and 53 known DEMs. Notably, the $b H L H$ TF Zm00001d043706 T001 was identified as a hub $(\mathrm{KME}=0.89, \mathrm{TOM}=0.22)$ gene in the module, which was trans-regulated by the IncRNA MSTRG.8888.1. Meanwhile, Zm00001d043706_ T001 have significant co-expression relationships with 73 PCTs, of which 19 contained 1-8 bHLH binding motifs (Table S10). The top five co-expressed mRNAs of Zm00001d043706_T001 included Zm00001d021761_ T001 (MYB-transcription factor 105, MYB105), Zm00001d028574_T001 (protein-serine/threonine phosphatase 14, PP2C14), Zm00001d028931_T003 (galactinol synthase 1, GOLS1), Zm00001d039685_T001 (raffinose synthase 1, RAFS1), and Zm00001d040190_ T001 (hydroxyproline-rich glycoprotein, HRGP) (Fig. 6B). These genes have been previously reported to correlate with the response of salt or other abiotic stress [41-44].

\section{Discussion}

In this study, two maize inbred lines (BML1234 and L2010-3) with contrasting salt tolerance were used for exploring the lncRNAs and their target transcripts involved in salt stress response. Our previous studies indicated that the two lines with different backgrounds showed different phenotypes under salt treatment of $150 \mathrm{mM} \mathrm{NaCl}$ concentration [28, 45]. Generally, the shoot and root growth was more seriously inhibited under salt treatment in the salt-sensitive line BML1234 compared with the salt-tolerant line L2010-3 [28]. A total of $178 \mathrm{~K}$ single nucleotide polymorphisms (SNPs) existed between the two lines [39]. In the present study, considerable variations in transcript expression levels were found between BML1234 and L2010-3, which coincided with the large genetic variations between the two lines. Salt stress was one of main abiotic stresses, which caused a major problem for plant growth and production. More and more evidence supported that lncRNAs play significant roles in stress response [41, 46-48]. Although some functional genes such as ZmNHX,ZmHTK, MIP, and SnRK2 have been proven to participate in the response to salt stress in plants $[6,7,49,50]$, only a few IncRNAs have been reported to involve salinity stress at a whole transcriptome level. To reveal the salt responsive lncRNAs and the mechanism underlying salt tolerance in maize, we first identified lncRNAs from maize seedling root at different salt treatment stages and normal conditions using whole transcriptome sequencing. Through a strict bioinformatic pipeline, we uncovered a total of 12,718 high-confidence lncRNAs. Compared with 
protein-coding genes, lncRNAs were shorter in length and had fewer exons in structures, which were consistent with the previous reports $[51,52]$. Then, we executed the comparative transcriptomic analysis, which identified more than 700 differential DELs between two lines with distinct salt tolerance. Most of the salt-responsive lncRNAs showed the differential expressions at the early stages of salt stress, especially in salt-sensitive line BML1234, which partially explained for the phenotypes of a more serious growth inhibition in BML1234.

The expression of functional genes was regulated by various factors, such as TFs, miRNAs, and lncRNAs [53-55]. As transcriptional regulators, lncRNAs affect the expression of functional genes directly or indirectly [56]. In the present study, we identified 45 cis- and 123 trans- lncRNA-mRNA pairs. Most of the lncRNA-mRNA pairs showed positive correlations in expression levels, whereas only 14 pairs (10 cis- and 4 trans-) had negative correlations. To further recognize the function of these DELs under salt stress, we performed KEGG pathway and GO term enrichment analysis for the target transcripts of the DELs. Some salt stress-responsive pathways and GO terms such as "oxidative phosphorylation" pathway and "calcium-transporting ATPase activity" term $[57,58]$ were significantly enriched with the target transcripts. These findings suggested that the DELs were involved in salt response of maize seedlings and contributed to the difference of salt tolerance between these two lines.

The previous studies showed that the interaction relationships between lncRNAs and TFs may ameliorate the expression levels of their target functional genes [59]. Therefore, we built a co-expression network to further investigate the relationships among the lncRNAs, TFs, and other mRNAs. In the lncRNA-TFsmRNA interaction networks, we found a total of 18 TFs were co-expressed with 5 lncRNAs and 56 mRNAs. Among the 5 lncRNAs, a hub lncRNA MSTRG.8888.1 acted as a trans-regulator and regulated the expression of another hub transcript bHLH TF Zm00001d043706_ T001. The $b H L H$ family was one of the largest families of transcription factors in plants [60], involved in plant response to diverse abiotic stresses, such as heavy metal toxicity, osmotic damages, drought, chilling, and salinity [61-63]. In this study, the top five functional mRNA co-expressed with Zm00001d043706_T001 contained Zm00001d021761_T001, Zm00001d028574_T001, Zm00001d028931_T003, Zm00001d039685_T001, and Zm00001d040190_T001. Zm00001d028931_T003 that encodes a galactinol synthase, which has been extensively reported to confer salt tolerance in plants by mediating the biosynthesis of galactinol and raffinose family oligosaccharides [41, 64]. Consistently, our present study found that the Zm00001d028931_T003 was significantly up-regulated under salt treatment with a higher expression level in the salt-tolerance line. Remarkably, the promoter of Zm00001d028931_T003 contained two G-box (CACG[TA]C) motifs, which are the typical $b H L H$ TF-binding motifs. This provided the evidence that $Z m 00001 d 028931 \_T 003$ was regulated by the bHLH TF Zm00001d043706_T001. Collectively, we constructed the regulatory network of salt-stress response, which was mediated by MSTRG.8888.1/ Zm00001d043706_T001. Some lncRNAs have been previously reported to act as miRNA targets or decoys, involving the regulation of gene expression [65]. Using a plant microRNA endogenous target mimics prediction tool, psMimic [66], five of these 742 DELs were identified as potential miRNA decoys and bound by six miRNAs, forming 12 miRNA-lncRNA duplexes (Table S11). In these pairs, each of the three DELs MSTRG.15598.1, MSTRG.15598.3, and MSTRG.7211.8 adsorbed the miRNAs zma-miR399b-5p, zmamiR399d-5p, and zma-miR399i-5p. Meanwhile, the DELs MSTRG.57825.1 and MSTRG.57690.7 acted as the sponges of one (zma-miR160d-3p) and two (zma$m i R 167 h-3 p$ and $z m a-m i R 167 i-3 p)$ miRNAs, respectively. Besides, using the miRbase (version 22.1) [67] and psRNATarget web server [68], we predicted the possible miRNA targets from the 742 DELs. In total, 322 lncRNAs were identified as the potential targets of 301 mature miRNAs (Table S12). Among them, the IncRNA MSTRG.8888.1 was distinguished as a possible target of $z m a-m i R 827-5 p$. Notably, $m i R 827$ has been extensively reported to regulate salt tolerance in plant species including cotton [69], banana [70], and Arabidopsis thaliana [71]. Based on these evidences, we present a model to summarize the putative regulatory pathway mediated by MSTRG.8888.1 (Fig. 7). As an upstream effector of this pathway, zma-miR827-5p responded to the signal of salt stress and regulated the MSTRG.8888.1 at the post-transcription level; then the changed MSTRG.8888.1 expression affected the transcription and translation of the $b H L H$; the altered protein abundance of the bHLH subsequently induced the upregulated expression of the five salt tolerance-related functional genes by binding their promoters (Fig. 7). Moreover, the differential responses of MSTRG.8888.1 to salt stress may partly account for the disparity in salt tolerance between the two lines (Fig. 7). The Snakemake workflow management system is a tool to create reproducible and scalable data analysis pipelines. Based on the Snakemake, we developed the NLncCirSmk to build an efficient, flexible, and reproducible bioinformatic analysis pipeline. The NLncCirSmk could deal 


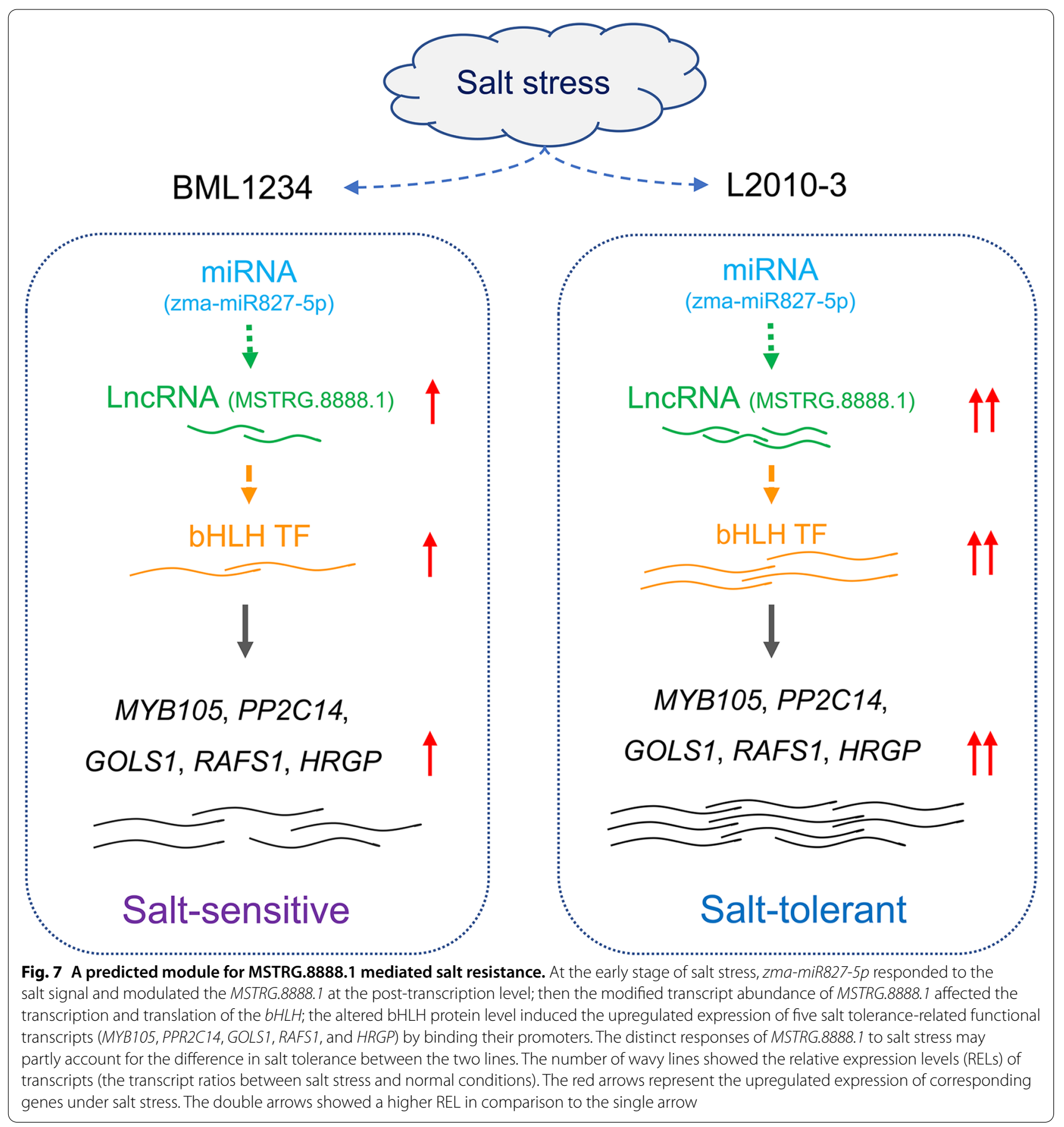

with numbers of samples at the same time with a modifiable profile. To reduce the false positives of lncRNAs identification, different approaches had been integrated into NLncCirSmk.

Collectively, our results provide new sights into further revelation of lncRNA function in maize tolerance to salt stress.

\section{Conclusions}

In conclusion, we identified 12,817 lncRNAs and 8,320 novel mRNAs in two maize lines with contrasting salt tolerance by using our developed bioinformatic pipeline. In total, 742 DELs were identified as the salt-tolerance transcripts. Among the five hub lncRNAs, MSTRG.8888.1 acted as a trans-regulatory and affected the expression of salt tolerance-relevant genes by targeting the 
bHLH transcript, Zm00001d043706_T001. Meanwhile, MSTRG.8888.1 was a potential target of miR827 that has been reported to involve the salt tolerance in other plant species. Based on these evidences, we present a model to summarize the putative regulatory pathway mediated by MSTRG.8888.1. Our results might expand our horizon for understanding the salt-tolerance mechanism regulated by lncRNAs in maize.

\section{Methods}

\section{Plant materials and salt treatment}

Two maize inbred lines, BML1234 (a salt-sensitive line) and L2010-3 (a salt-tolerant line), were used in this study, which have been described in our previous study [29]. For each line, the seeds were surface-sterilized using $10 \%$ (v/v) $\mathrm{H}_{2} \mathrm{O}_{2}$ for $15 \mathrm{~min}$ and then rinsed three times with distilled water. After that, the seeds were germinated on filter paper saturated with distilled water and then grown at $26^{\circ} \mathrm{C}$ under 14 -h day $/ 10$-h night conditions.

Uniform seedlings with two leaves were randomly divided into two groups: CK (cultivated in Hoagland's solutions) and salt treatment (cultivated in Hoagland's solutions with $150 \mathrm{mM} \mathrm{NaCl}$ ). These seedlings were then cultured in a growth room with a relative humidity of $70 \%$ at $26^{\circ} \mathrm{C}$, and a cycle of 14 -h day $/ 10$-h night. At 0 , 6,18 , and $36 \mathrm{~h}$, the mixed roots from three seedlings of each line were individually collected as the samples for whole transcriptome sequencing, with two biological repetitions.

\section{RNA isolation, sequencing, and quality control}

Referring to the manufacturer's instructions, total RNA of each sample was extracted using the HiPure Plant RNA Maxi Kit (Magen Company, Guangzhou). The quality and purity of RNA were analyzed with a 2100 Bioanalyzer and RNA 6000 Nano kit 5067-1511 (Agilent, CA, United S). Ribosome RNA (rRNA) was filtered by Illumina Ribo-Zero rRNA Removal Kit. RNA libraries were constructed by the Illumina sequencing platform and sequenced on a Hiseq 4000 system (Illumina) using the PE150 method. All raw data were deposited in Genome Sequence Archive (GSA) in National Genomics Data Center (NGDC) database with the accession number CRA003872.

Raw reads were filtered with fastp [72] to remove the low-quality reads, polyN-containing reads and adapter reads. Using bowtie2 [73], the remaining high-quality reads were further aligned in the plant rRNA database (RNACentral v16) [74] to remove rRNA sequences.

\section{Genome alignment, isoform assembly and isoform expression calculation}

After filtering rRNA, the remaining reads were aligned to the maize genome (B73 RefGen_v4) using hisat2 [75], allowing up to 2 mismatches. Then, we assembled the transcriptome using stringtie program with params '-m 200 -a 10 -conservative - $\left.50-\mathrm{u}^{\prime}\right)$. Subsequently, the program "stringtie merge" (params: -m 200 -c 3) was used to merge the information of all transcripts, generating the integrated transcript information [76]. The expression level of each isoform was calculated by re-assembling with integrated transcript information. The read counts and transcripts per million (TPM) matrixes were directly extracted from the files generated by a Python script (prepDE.py, provided by stringtie program). After filtering the low-expression transcripts by a customized $\mathrm{R}$ script, the counts matrix was used to perform differential expression analysis and the TPM matrix was used to conduct correlation analysis, principal component analysis (PCA), and co-expression network construction.

\section{Identification of pseudo IncRNAs and novel protein-coding transcripts}

To identify putative lncRNAs and novel PCTs, we used a rigorous set of criteria to annotate the assembled transcripts. First, a flexible extraction of long non-coding RNAs tool (FEELnc) was employed to detect potential lncRNAs based on a random forest model [77]. Then, the integrated transcripts were compared with the maize reference transcripts by the gffcompare program [78]. The following steps were performed to identify the lncRNAs from the transcripts based on their characteristics: (1) transcripts with class_code of "i", "u", "x" and "o" were selected; (2) transcripts with a length $<200 \mathrm{bp}$ and an exon count $<2$ were removed; (3) transcripts with a $\mathrm{TPM} \geq 1$ were selected; (4) transcripts that did not pass the protein-coding-score test were eliminated using the Coding Potential Calculator (CPC version2) [79] and Coding-Non-Coding Index (CNCI) [80]; (5) known mRNA and transcripts with protein-coding domain in Pfam databases were removed [81]. The intersections of non-coding transcripts identified by Pfam, CNCI, CPC and FEElnc were considered as the putative lncRNAs.

Additionally, we used the transcripts with class_code of " $\mathrm{j}$ " and " $u$ " for the prediction of novel protein-coding transcripts. After predicting candidate coding regions within transcripts by TransDecoder software (https:// github.com/TransDecoder), we calculated the coding potential score of each transcript using $\mathrm{CPC} 2$, CNCI, PfamScan, and an alignment-free method Coding Potential Assessment Tool (CPAT) [82]. Those transcripts that were simultaneously defined as coding mRNAs by four methods were recognized as novel mRNAs. The Clusters 
of Orthologous Genes (COG) categories and functional annotations were then predicted using an online tool eggnog-mapper (http://eggnog-mapper.embl.de/) [83].

\section{Correlation analysis, PCA, and differential expression analysis}

To evaluate the relationship between samples, we performed the correlation analysis and PCA for the 28 samples. First, we filtered out the transcripts with lower expression levels, which may be caused by assembly errors. Then, the correlation analysis and PCA were carried out with the cor function using the Pearson method and prcomp packages in R, respectively. Finally, Deseq2 were used to detect differentially expressed lncRNAs and novel protein-coding transcripts. Transcripts with $|\log 2 \mathrm{FC}|>2$ and $\mathrm{FDR}<0.01$ were identified as significant DETs [84]. A Perl script was used to fetch and count the DETs in the different comparison groups.

\section{Prediction of IncRNA targets}

To determine the cis-target transcripts of lncRNAs, we searched for PCTs within $100 \mathrm{~Kb}$ upstream and $100 \mathrm{~Kb}$ downstream of the lncRNAs [53]. The Pearson correlation coefficient (PCC) between the IncRNA and the corresponding PCT was then calculated based on their expression levels. The PCTs that met the strict standards $(|\mathrm{PCC}|>0.6, P<0.05)$ were considered as cis-target transcripts of the lncRNAs.

Furthermore, we used the LncTar program to predict the trans-targets of lncRNAs based on complementary base pairing [85]. The transcript was considered a transtarget of the lncRNA, when the free energy of pairing sites between transcript and lncRNA was lower than the threshold of standardized free energy (ndG< -0.2) [51]. Besides, the PCC between the lncRNA and the corresponding transcript was calculated. Those mRNAs with $\mid$ PCC $\mid>0.8$ and $P$-value $<0.01$ in IncRNA-mRNA pairs were defined as the putative trans-target mRNAs of the lncRNAs [86].

\section{Identification of transcription factors}

TFs have been proved to play crucial roles in maize response to salt stress [87]. In the present study, an online tool PlantTFcat [88] was used to conduct TF analysis for all the DETs.

\section{Weighted gene co-expression network construction}

The WGCNA was executed with the WGCNA (v1.69) package in $R$ [89] based on the normalized expressions of DETs. The transcripts with the eigengene connectivity $(\mathrm{KME})>0.75$ or $<-0.75$ and topological overlap measure $(\mathrm{TOM})>0.2$ were defined as the hub transcripts.

\section{Quantitative real-time PCR}

To verify the sequencing results, we randomly selected six transcripts for qRT-PCR. The primer pairs for qRTPCR were designed using the Primer 5.0 software and are shown in Table S3. The qRT-PCR was carried out with an Applied Biosystems 7500 Real-Time PCR System with three biological repetitions. The reaction program was as follows: $2 \mathrm{~min}$ at $98{ }^{\circ} \mathrm{C}, 2 \mathrm{~s}$ at $98{ }^{\circ} \mathrm{C}, 10 \mathrm{~s}$ at $59^{\circ} \mathrm{C}, 40$ cycles. A thermal denaturing step was then performed for generation of the melting curves for amplification specificity verification. The maize Actin 1 gene (Zm00001d010159) was selected as the reference for normalizing the gene expression. The $2^{-\triangle \triangle \mathrm{ct}}$ method was used for calculating the relative expression levels of target genes.

\section{KEGG Pathway and GO term enrichment analysis}

Gene Ontology (GO) and Kyoto Encyclopedia of Genes and Genomes (KEGG) pathway enrichment analyses were performed using the OmicShare, a free online platform for data analysis (www.omicshare.com/tools).

\section{Construction of regulatory network}

In the module enriched with the transcripts involving abiotic stress-response and transferase activity, we combined the hub lncRNAs, the corresponding target transcripts, and the other transcripts in the module and constructed the regulation network mediated by the hub lncRNAs. Cytoscape 3.7.1 [90] was then utilized to draw the putative interaction network.

\section{Statements}

The two maize lines used in this study were provided by Sichuan Agricultural University and comply with relevant institutional, national, and international guidelines and legislation.

\section{Abbreviations \\ bHLH: Basic helix-loop helix protein; bZIP: Basic leucine zipper; CNCl: Coding- Non-Coding Index; CPC: Coding Potential Calculator; DELs: Differentially expressed IncRNAs; DEMs: Differentially expressed mRNAs; DETs: Differentially expressed transcripts; FDR: False discovery rate; FTs: Functional transcripts; GO: Gene ontology; KEGG: Kyoto Encyclopedia of Genes and Genomes; KME: Eigengene connectivity; lincRNAs: Long intergenic ncRNAs; LncRNAs: Long non-coding RNAs; mRNAs: Messenger RNAs; ORF: Open reading frame; PCA: Principal component analysis; PCC: Pearson correlation coefficient; PCs: Principal components; PCTs: Protein coding transcripts; qRT-PCR: Quantitative real-time PCR; RNA-seq: RNA sequencing; ROS: Reactive oxygen species; TFs: Transcription factors; TOM: Topological overlap measure; WGCNA: Weighted gene co-expression network analysis.}

\section{Supplementary Information}

The online version contains supplementary material available at https://doi. org/10.1186/s12864-021-08286-7. 
Additional file 1: Figure S1. COGs annotation for novel mRNAs. Different alphabets indicate various COGs. Detailed information of COGs can be found in the COG database (https://www.ncbi.n/m.nih.gov/research/cog/). Figure S2. qRT-PCR validation of identified IncRNAs and PCTs. Figure S3. Venn diagrams for DELs in different comparable groups. Figure S4. Determination of soft thresholding power in the WGCNA. The left panel shows the influence of soft threshold power on the scale free topological fit index; the right panel shows the influence of soft threshold power on the average connectivity

Additional file 2: Table S1. Sequencing and mapping statistics for each sample. Table S2. Annotations of novel mRNAs. Table S3. Primer sequences for qRT-PCR. Table S4. Predicted TFs in the DEMs. Table S5. Cis-target transcripts of DELs. Table S6. Trans-target transcripts of DELs. Table S7. KEGG enrichment analysis for target mRNAs. Table S8. GO enrichment analysis for target mRNAs. Table S9. Expressions of DETs for WGCNA. Table S10. The 19 co-expressed genes of the bHLHTF, which contained bHLH binding motifs. Table S11. Prediction of IncRNA acting as miRNA decoys. Table S12. Identification of DELs acting as miRNA targets.

\section{Acknowledgements}

We thanks to other graduate students who attended this project. We also thank the Maize Research Institute of Sichuan Agricultural University for providing the platform.

\section{Authors' contributions}

YS conceived the project and designed the experiments. PL, YZ, and CZ performed the experiments. PL conducted the data analysis. CY and LM participated in some of the experiments. PL and YS drafted the manuscript with the contribution from GP. All the authors reviewed and approved the final manuscript.

\section{Funding}

This work is supported by the National Nature Science Foundation of China (31871637 and 32072073), the Sichuan Science and Technology Program (2021 JDTD0004 and 2021YJ0476).

\section{Availability of data and materials}

The reference genome and genes of maize are available from RefGen_V4 (http://www.gramene.org/). The datasets generated during the current study are available in the Genome Sequence Archive (GSA) in National Genomics Data Center, China National Center for Bioinformation / Beijing Institute of Genomics, Chinese Academy of Sciences, under accession number CRA003872 that are publicly accessible at https://ngdc.cncb.ac.cn/gsa.

\section{Declarations}

Ethics approval and consent to participate

Not applicable

\section{Consent for publication}

Not applicable

\section{Competing interests}

The authors declare that they have no competing interests

Received: 7 September 2021 Accepted: 29 December 2021

Published online: 13 January 2022

\section{References}

1. Hanin M, Ebel C, Ngom M, Laplaze L, Masmoudi K. New Insights on Plant Salt Tolerance Mechanisms and Their Potential Use for Breeding. Front Plant Sci. 2016;7:1787. https://doi.org/10.3389/fpls.2016.01787.

2. Munns R, Tester M. Mechanisms of Salinity Tolerance. Annu Rev Plant Biol. 2008:59:651-81.

3. Ahanger MA, Tomar NS, Tittal M, Argal S, Agarwal RM. Plant growth under water/salt stress: ROS production; antioxidants and significance of added potassium under such conditions. Physiol Mol Biol Plants. 2017;23:731-44.

4. Pang C-H, Wang B-S. Oxidative Stress and Salt Tolerance in Plants. In: Lüttge U, Beyschlag W, Murata J, editors. Progress in Botany. Berlin, Heidelberg: Springer; 2008. p. 231-45. https://doi.org/10.1007/ 978-3-540-72954-9_9.

5. Faroog M, Hussain M, Wakeel A, Siddique KHM. Salt stress in maize: effects, resistance mechanisms, and management. A review Agron Sustain Dev. 2015:35:461-81.

6. Huang Y, Zhang X, Li Y, Ding J, Du H, Zhao Z, et al. Overexpression of the Suaeda salsa SsNHX1 gene confers enhanced salt and drought tolerance to transgenic Zea mays. J Integr Agric. 2018;17:2612-23.

7. Zhang F, Chen X, Wang J, Zheng J. Overexpression of a maize SNF-related protein kinase gene, $Z \mathrm{mSnRK} 2.11$ reduces salt and drought tolerance in Arabidopsis. J Integr Agric. 2015;14:1229-41.

8. Zhang $M$, Cao $Y$, Wang Z, Wang Z, Shi J, Liang $X$, et al. A retrotransposon in an HKT1 family sodium transporter causes variation of leaf $\mathrm{Na}+$ exclusion and salt tolerance in maize. New Phytol. 2018;217:1161-76.

9. Zhang M, Liang X, Wang L, Cao Y, Song W, Shi J, et al. A HAK family Na + transporter confers natural variation of salt tolerance in maize. Nat Plants. 2019;5:1297-308

10. Wu J, Jiang $Y$, Liang $Y$, Chen L, Chen W, Cheng B. Expression of the maize MYB transcription factor ZmMYB3R enhances drought and salt stress tolerance in transgenic plants. Plant Physiol Biochem. 2019;137:179-88.

11. Cao H, Wang L, Nawaz MA, Niu M, Sun J, Xie J, et al. Ectopic Expression of Pumpkin NAC Transcription Factor CmNAC1 Improves Multiple Abiotic Stress Tolerance in Arabidopsis. Front Plant Sci. 2017:8:2052. https://doi. org/10.3389/fpls.2017.02052.

12. Li H, Gao Y, Xu H, Dai Y, Deng D, Chen J. ZmWRKY33, a WRKY maize transcription factor conferring enhanced salt stress tolerances in Arabidopsis. Plant Growth Regul. 2013;70:207-16.

13. Li M, Chen R, Jiang Q, Sun X, Zhang H, Hu Z. GmNAC06, a NAC domain transcription factor enhances salt stress tolerance in soybean. Plant Mol Biol. 2021;105:333-45.

14. Shen Z, Ding M, Sun J, Deng S, Zhao R, Wang M, et al. Overexpression of PeHSF mediates leaf ROS homeostasis in transgenic tobacco lines grown under salt stress conditions. Plant Cell Tissue Organ Cult PCTOC. 2013:115:299-308.

15. Ying S, Zhang D-F, Fu J, Shi Y-S, Song Y-C, Wang T-Y, et al. Cloning and characterization of a maize bZIP transcription factor, ZmbZIP72, confers drought and salt tolerance in transgenic Arabidopsis. Planta. 2012:235:253-66

16. Mahajan S, Pandey GK, Tuteja N. Calcium- and salt-stress signaling in plants: Shedding light on SOS pathway. Arch Biochem Biophys. 2008:471:146-58

17. Yang Y, Guo Y. Unraveling salt stress signaling in plants. J Integr Plant Biol. 2018;60:796-804

18. You J, Chan Z. ROS Regulation During Abiotic Stress Responses in Crop Plants. Front Plant Sci. 2015;6:1092. https://doi.org/10.3389/fpls.2015. 01092.

19. Zhao X, Bai X, Jiang C, Li Z. Phosphoproteomic Analysis of Two Contrasting Maize Inbred Lines Provides Insights into the Mechanism of SaltStress Tolerance. Int J Mol Sci. 2019;20:1886.

20. Statello L, Guo C-J, Chen L-L, Huarte M. Gene regulation by long non-coding RNAs and its biological functions. Nat Rev Mol Cell Biol. 2021;22:96-118.

21. Bazin J, Baerenfaller K, Gosai SJ, Gregory BD, Crespi M, BaileySerres J. Global analysis of ribosome-associated noncoding RNAs unveils new modes of translational regulation. Proc Natl Acad Sci. 2017;114:E10018-27

22. Mao Y, Xu J, Wang Q, Li G, Tang X, Liu T, et al. A natural antisense transcript acts as a negative regulator for the maize drought stress response gene ZmNAC48. J Exp Bot. 2021. https://doi.org/10.1093/jxb/erab023.

23. Ponting CP, Oliver PL, Reik W. Evolution and Functions of Long Noncoding RNAs. Cell. 2009;136:629-41.

24. Li L, Eichten SR, Shimizu R, Petsch K, Yeh C-T, Wu W, et al. Genome-wide discovery and characterization of maize long non-coding RNAs. Genome Biol. 2014;15:R40.

25. Huanca-Mamani W, Arias-Carrasco R, Cárdenas-Ninasivincha S, RojasHerrera M, Sepúlveda-Hermosilla G, Caris-Maldonado JC, et al. Long 
Non-Coding RNAs Responsive to Salt and Boron Stress in the Hyper-Arid Lluteño Maize from Atacama Desert. Genes. 2018;9:170.

26. Lv Y, Hu F, Zhou Y, Wu F, Gaut BS. Maize transposable elements contribute to long non-coding RNAs that are regulatory hubs for abiotic stress response. BMC Genomics. 2019;20:864.

27. Köster J, Rahmann S. Snakemake-a scalable bioinformatics workflow engine. Bioinformatics. 2012;28:2520-2.

28. Zhang X, Liu P, Qing C, Yang C, Shen Y, Ma L. Comparative transcriptome analyses of maize seedling root responses to salt stress. PeerJ. 2021;9:e10765.

29. Jiao Y, Peluso P, Shi J, Liang T, Stitzer MC, Wang B, et al. Improved maize reference genome with single-molecule technologies. Nature. 2017:546:524-7.

30. Yan Q, Wu F, Yan Z, Li J, Ma T, Zhang Y, et al. Differential co-expression networks of long non-coding RNAs and mRNAs in Cleistogenes songorica under water stress and during recovery. BMC Plant Biol. 2019;19:23.

31. Fan T, Zhang Q, Hu Y, Wang Z, Huang Y. Genome-wide identification of IncRNAs during hickory (Carya cathayensis) flowering. Funct Integr Genomics. 2020;20:591-607.

32. Fu L, Ding Z, Tan D, Han B, Sun X, Zhang J. Genome-wide discovery and functional prediction of salt-responsive IncRNAs in duckweed. BMC Genomics. 2020;21:212.

33. Bai J, Sun F, Wang M, Su L, Li R, Caetano-Anollés G. Genome-wide analysis of the MYB-CC gene family of maize. Genetica. 2019;147:1-9.

34. Cai R, Dai W, Zhang C, Wang Y, Wu M, Zhao Y, et al. The maize WRKY transcription factor ZmWRKY17 negatively regulates salt stress tolerance in transgenic Arabidopsis plants. Planta. 2017:246:1215-31.

35. Han G, Lu C, Guo J, Qiao Z, Sui N, Qiu N, et al. C2H2 Zinc Finger Proteins Master Regulators of Abiotic Stress Responses in Plants. Front Plant Sci. 2020;1 1:115. https://doi.org/10.3389/fpls.2020.00115.

36. Lee S, Lee J, Paek K-H, Kwon S-Y, Cho HS, Kim SJ, et al. A novel WD40 protein, BnSWD1, is involved in salt stress in Brassica napus. Plant Biotechnol Rep. 2010;4:165-72.

37. Li Z, Liu C, Zhang Y, Wang B, Ran Q, Zhang J. The bHLH family member ZmPTF1 regulates drought tolerance in maize by promoting root development and abscisic acid synthesis. J Exp Bot. 2019;70:5471-86.

38. Han F, Li J, Zhao R, Liu L, Li L, Li Q, et al. Identification and co-expression analysis of long noncoding RNAs and mRNAs involved in the deposition of intramuscular fat in Aohan fine-wool sheep. BMC Genomics. 2021;22:98.

39. Ma L, Zhang M, Chen J, Qing C, He S, Zou C, et al. GWAS and WGCNA uncover hub genes controlling salt tolerance in maize (Zea mays L seedlings. Theor Appl Genet. 2021. https://doi.org/10.1007/ s00122-021-03897-w.

40. Wang P, Dai L, Ai J, Wang Y, Ren F. Identification and functional prediction of cold-related long non-coding RNA (IncRNA) in grapevine. Sci Rep. 2019;9:6638.

41. Hu S, Zhang M, Yang Y, Xuan W, Zou Z, Arkorful E, et al. A novel insight into nitrogen and auxin signaling in lateral root formation in tea plant [Camellia sinensis L. O. Kuntze]. BMC Plant Biol. 2020;20:232.

42. Liu L, Wu X, Sun W, Yu X, Demura T, Li D, et al. Galactinol synthase confers salt-stress tolerance by regulating the synthesis of galactinol and raffinose family oligosaccharides in poplar. Ind Crops Prod. 2021:165:113432.

43. Xing B, Gu C, Zhang T, Zhang Q, Yu Q, Jiang J, et al. Functional Study of BpPP2C1 Revealed Its Role in Salt Stress in Betula platyphylla. Front Plant Sci. 2021;11:617635.

44. Zagorchev L, Kamenova P, Odjakova M. The Role of Plant Cell Wall Proteins in Response to Salt Stress. Sci World J. 2014;2014:e764089.

45. Gao Y, Lu Y, Wu M, Liang E, Li Y, Zhang D, et al. Ability to Remove Na+ and Retain K+ Correlates with Salt Tolerance in Two Maize Inbred Lines Seedlings. Front Plant Sci. 2016;7:1716. https://doi.org/10.3389/fpls.2016. 01716.

46. Sun X, Zheng H, Li J, Liu L, Zhang X, Sui N. Comparative Transcriptome Analysis Reveals New IncRNAs Responding to Salt Stress in Sweet Sorghum. Front Bioeng Biotechnol. 2020;8:331. https://doi.org/10.3389/ fbioe.2020.00331.

47. Wen $X$, Ding $Y$, Tan Z, Wang J, Zhang $D$, Wang $Y$. Identification and characterization of cadmium stress-related LncRNAs from Betula platyphylla. Plant Sci. 2020;299:110601.
48. Zhang $X$, Dong J, Deng F, Wang W, Cheng Y, Song L, et al. The long noncoding RNA IncRNA973 is involved in cotton response to salt stress. BMC Plant Biol. 2019:19:459.

49. Zhu C, Schraut D, Hartung W, Schäffner AR. Differential responses of maize MIP genes to salt stress and ABA. J Exp Bot. 2005;56:2971-81.

50. Zörb C, Noll A, Karl S, Leib K, Yan F, Schubert S. Molecular characterization of $\mathrm{Na}+/ \mathrm{H}+$ antiporters $(\mathrm{ZmNHX}$ ) of maize (Zea mays $\mathrm{L}$.) and their expression under salt stress. J Plant Physiol. 2005;162:55-66.

51. Pang J, Zhang $X, M a X$, Zhao J. Spatio-Temporal Transcriptional Dynamics of Maize Long Non-Coding RNAs Responsive to Drought Stress. Genes. 2019;10:138.

52. Wang H, Niu Q-W, Wu H-W, Liu J, Ye J, Yu N, et al. Analysis of non-coding transcriptome in rice and maize uncovers roles of conserved IncRNAs associated with agriculture traits. Plant J. 2015;84:404-16.

53. Augustino SMA, Xu Q, Liu X, Mi S, Shi L, Liu Y, et al. Integrated analysis of IncRNAs and mRNAs reveals key trans-target genes associated with ETECF4ac adhesion phenotype in porcine small intestine epithelial cells. BMC Genomics. 2020;21:780.

54. Ding D, Zhang L, Wang H, Liu Z, Zhang Z, Zheng Y. Differential expression of miRNAs in response to salt stress in maize roots. Ann Bot. 2009;103:29-38.

55. Zhang Y, Ge F, Hou F, Sun W, Zheng Q, Zhang X, et al. Transcription Factors Responding to Pb Stress in Maize. Genes. 2017;8:231.

56. Long $Y$, Wang $X$, Youmans DT, Cech TR. How do IncRNAs regulate transcription? Sci Adv. 2017;3:eaao2110.

57. Braun H-P. The Oxidative Phosphorylation system of the mitochondria in plants. Mitochondrion. 2020;53:66-75.

58. AK Srivastava AN Rai VY Patade P Suprasanna Calcium Signaling and Its Significance in Alleviating Salt Stress in Plants P Ahmad MM Azooz MNV Prasad Salt Stress in Plants Signalling Omics and Adaptations Springer New York 2013197218 https://doi.org/10.1007/ 978-1-4614-6108-1_9

59. Wang B, Tang D, Zhang Z, Wang Z. Identification of aberrantly expressed IncRNA and the associated TF-mRNA network in hepatocelIular carcinoma. J Cell Biochem. 2020;121:1491-503.

60. Pireyre M, Burow M. Regulation of MYB and bHLH Transcription Factors: A Glance at the Protein Level. Mol Plant. 2015;8:378-88.

61. Dong Y, Wang C, Han X, Tang S, Liu S, Xia X, et al. A novel bHLH transcription factor PebHLH35 from Populus euphratica confers drought tolerance through regulating stomatal development, photosynthesis and growth in Arabidopsis. Biochem Biophys Res Commun. 2014:450:453-8.

62. Jiang Y, Yang B, Deyholos MK. Functional characterization of the Arabidopsis bHLH92 transcription factor in abiotic stress. Mol Genet Genomics. 2009;282:503-16

63. Yadav BS, Mani A. Analysis of bHLH coding genes of Cicer arietinum during heavy metal stress using biological network. Physiol Mol Biol Plants. 2019;25:113-21.

64. Nishizawa A, Yabuta Y, Shigeoka S. Galactinol and Raffinose Constitute a Novel Function to Protect Plants from Oxidative Damage. Plant Physiol. 2008;147:1251-63.

65. LvY, Liang Z, Ge M, Qi W, Zhang T, Lin F, et al. Genome-wide identification and functional prediction of nitrogen-responsive intergenic and intronic long non-coding RNAs in maize (Zea mays L.). BMC Genomics. 2016:17:350.

66. Wu H-J, Wang Z-M, Wang M, Wang X-J. Widespread Long Noncoding RNAs as Endogenous Target Mimics for MicroRNAs in Plants. Plant Physiol. 2013;161:1875-84.

67. Kozomara A, Birgaoanu M, Griffiths-Jones S. miRBase: from microRNA sequences to function. Nucleic Acids Res. 2019;47:D155-62.

68. Dai X, Zhuang Z, Zhao PX. psRNATarget: a plant small RNA target analysis server (2017 release). Nucleic Acids Res. 2018;46:W49-54.

69. Prasad Shaw B. Salt stress tolerance in plants: the role of miRNAs. Adv Plants Agric Res. 2018:8(6):411-5. https://doi.org/10.15406/apar.2018.08. 00360.

70. Lee WS, Gudimella R, Wong GR, Tammi MT, Khalid N, Harikrishna JA. Transcripts and MicroRNAs Responding to Salt Stress in Musa acuminata Colla (AAA Group) cv. Berangan Roots. PLOS ONE. 2015;10:e0127526.

71. Shukla PS, Borza T, Critchley AT, Hiltz D, Norrie J, Prithiviraj B. Ascophyllum nodosum extract mitigates salinity stress in Arabidopsis thaliana by 
modulating the expression of miRNA involved in stress tolerance and nutrient acquisition. PLOS ONE. 2018;13:e0206221.

72. Chen S, Zhou Y, Chen Y, Gu J. fastp: an ultra-fast all-in-one FASTQ preprocessor. Bioinformatics. 2018;34:1884-90.

73. Langmead B, Salzberg SL. Fast gapped-read alignment with Bowtie 2. Nat Methods. 2012;9:357-9.

74. RNAcentral Consortium T, Sweeney BA, Petrov Al, Burkov B, FinnBateman RDA, et al. RNAcentral: a hub of information for non-coding RNA sequences. Nucleic Acids Res. 2019;47:D221-9.

75. Kim D, Langmead B, Salzberg SL. hisAt: a fast spliced aligner with low memory requirements. Nat Methods. 2015;12:357.

76. Pertea M, Pertea GM, Antonescu CM, Chang T-C, Mendell JT, Salzberg SL. StringTie enables improved reconstruction of a transcriptome from RNAseq reads. Nat Biotechnol. 2015;33:290-5.

77. Wucher V, Legeai F, Hédan B, Rizk G, Lagoutte L, Leeb T, et al. FEELnc: a tool for long non-coding RNA annotation and its application to the dog transcriptome. Nucleic Acids Res. 2017;45:e57-e57.

78. Pertea G, Pertea M, GFF Utilities. GffRead and GffCompare. F1000Research. 2020;9:304.

79. Kang Y-J, Yang D-C, Kong L, Hou M, Meng Y-Q, Wei L, et al. CPC2: a fast and accurate coding potential calculator based on sequence intrinsic features. Nucleic Acids Res. 2017;45:W12-6.

80. Sun L, Luo H, Bu D, Zhao G, Yu K, Zhang C, et al. Utilizing sequence intrinsic composition to classify protein-coding and long non-coding transcripts. Nucleic Acids Res. 2013;41:e166-e166.

81. Madeira F, Park Y mi, Lee J, Buso N, Gur T, Madhusoodanan N, et al. The EMBL-EBI search and sequence analysis tools APIs in 2019. Nucleic Acids Res. 2019;47:W636-41.

82. Wang L, Park HJ, Dasari S, Wang S, Kocher J-P, Li W. CPAT: Coding-Potential Assessment Tool using an alignment-free logistic regression model. Nucleic Acids Res. 2013:41:e74.

83. Huerta-Cepas J, Szklarczyk D, Heller D, Hernández-PlazaForslund ASK, Cook H, et al. eggNOG 5.0: a hierarchical, functionally and phylogenetically annotated orthology resource based on 5090 organisms and 2502 viruses. Nucleic Acids Res. 2019;47:D309-14.

84. Love MI, Huber W, Anders S. Moderated estimation of fold change and dispersion for RNA-seq data with DESeq2. Genome Biol. 2014;15:550.

85. Li J, Ma W, Zeng P, Wang J, Geng B, Yang J, et al. LncTar: a tool for predicting the RNA targets of long noncoding RNAs. Brief Bioinform. 2015;16:806-12.

86. Shao J, Zhang Y, Fan G, Xin Y, Yao Y. Transcriptome analysis identified a novel 3-LncRNA regulatory network of transthyretin attenuating glucose induced hRECs dysfunction in diabetic retinopathy. BMC Med Genomics. 2019:12:134.

87. Yoon Y, Seo DH, Shin H, Kim HJ, Kim CM, Jang G. The Role of StressResponsive Transcription Factors in Modulating Abiotic Stress Tolerance in Plants. Agronomy. 2020;10:788.

88. Jin J, Tian F, Yang D-C, Meng Y-Q, Kong L, Luo J, et al. PlantTFDB 4.0: toward a central hub for transcription factors and regulatory interactions in plants. Nucleic Acids Res. 2017;45:D1040-5.

89. Langfelder P, Horvath S. WGCNA: an R package for weighted correlation network analysis. BMC Bioinformatics. 2008;9:559.

90. Shannon P, Markiel A, Ozier O, Baliga NS, Wang JT, Ramage D, et al. Cytoscape: A Software Environment for Integrated Models of Biomolecular Interaction Networks. Genome Res. 2003;13:2498-504.

\section{Publisher's Note}

Springer Nature remains neutral with regard to jurisdictional claims in published maps and institutional affiliations.

Ready to submit your research? Choose BMC and benefit from:

- fast, convenient online submission

- thorough peer review by experienced researchers in your field

- rapid publication on acceptance

- support for research data, including large and complex data types

- gold Open Access which fosters wider collaboration and increased citations

- maximum visibility for your research: over $100 \mathrm{M}$ website views per year

At $\mathrm{BMC}$, research is always in progress.

Learn more biomedcentral.com/submissions 